\title{
Calculation of the Optimal Doses of the Ameliorant Neutralizing Heavy Metals in Anthropogenically Polluted Soils
}

\author{
S.J. Tamoyan \\ Armenian National Agrarian University \\ samveltamoyang@gmail.com
}

\section{ART I CLE INFO}

Keywords:

heavy metals, ameliorant, absorbing surface, grain size, dose

\begin{abstract}
A B S T R A C T
The paper considers improvement ways of the technology for neutralizing soil polluting heavy metals. The common technologies do not yet specify the effective and environmentally friendly application doses of ameliorants absorbing the pollutants.

The theoretical calculations have proved that there is a quantitative relationship between the sizes of different fractions contained in the ameliorant and the optimal share of the ameliorant, in accordance wih the level of soil pollution. The derived relation enables to calculate the optimal shares of the ameliorants absorbing heavy metals in soil.
\end{abstract}

\section{Introduction}

One of the most important agroecological issues refers to the pollution of soil with heavy metals that not noly decreases its productivity, but also worsens the quality of the products, thus endangering the human health. Armenia is among the countries facing this problem, with the content of pollutants being 5-40 times the size of the baseline values in about 55000 hectares of land area polluted with heavy metals (in Tavush, Lori, Syunik, Ararat and other marzes) (Amirjanyan, 1993). Therefore, reclamation of these soils is critical.

\section{Materials and methods}

The most practical way to reclaim soils polluted with heavy metals is neutralization of the excess pollution elements in the soil. The main approach is considered to be enrichment of the soil medium with ecologically pure ameliorants substances that would either absorb the heavy metals or turn them into compounds that will be inaccessible for plants, thus reducing the opportunity to penetrate into the plant (Hayrapetyan, et al, 2008, 2009, Galstyan, et al, 2015).

However, from the standpoint of the soil's ecological purity requirement, the doses of the ameliorants entered into the soil (zeolite, dacite tuff) should be also taken control of, even if they have a natural origin and are considered ecologically clean substances. The doses of their use are still to be adjusted: based on the pollutants and the level of soil pollution, the optimal amount of an ameliorant should 
be selected so that it ensures the best absorption with the minimum dose and the best grain size proportion.

In a theoretical work (Tamoyan, 2013) published on this subject, calculation of the ameliorant optimized mass and the grain size was performed based on a certain value of the grain size. However, the ameliorant used in the commercial production is actually a mixture of fractions with different grain sizes; therefore, this calculation needs to be improved.

This article considers such a generalization with the help of a simple mathematical calculation on the basis of the summability property of surfaces and masses.

\section{Results and discussions}

The amount of ameliorant to be applied to the soil to neutralize the polluting amount of heavy metals depends on a number of factors, including the content (expressed in percentage or in shares) of fractions with different grain size in the ameliorant. The ameliorant applied (zeolite, dacite tuff) is, as a matter of fact, a mixture of fractions with $0.1 \mathrm{~mm}-5 \mathrm{~mm}$ grain size.

The share of the grain size mass in this mixture can be presented with the help of the whole mass of the ameliorant: $m_{1}=A \cdot m_{a m}, \quad m_{2}=B \cdot m_{a m}, \quad \ldots m_{i}=N \cdot m_{a m}$, where $A, B, \ldots N$ is the share of the particular fraction in the ameliorant.

With the help of the expression $S_{a m}=3 \cdot \frac{m_{a m}}{\rho_{a m} \cdot d_{a m}}$ (Tamoyan, 2013) obtained for the absorption surface of separate grain size, the contribution of all grain sizes existing in the ameliorant can be calculated, while with their sum, the contribution of the whole ameliorant in absorption of heavy metals can be calculated.

At the first approximation, the efficient heavy metalabsorbing surface created by the whole ameliorant in the soil can be expressed in the form of the sum of surfaces created by different grain sizes. Mathematically, this can be presented by the following sum:

$$
\begin{gathered}
S_{a m}=S_{1}+S_{2}+\ldots+S_{i}= \\
=3 \cdot \frac{m_{1}}{\rho_{a m} \cdot d_{1}}+3 \cdot \frac{m_{2}}{\rho_{a m} \cdot d_{2}}+\ldots+3 \cdot \frac{m_{i}}{\rho_{a m} \cdot d_{i}}= \\
=3 \cdot \frac{m_{a m}}{\rho_{a m}} \cdot\left(\frac{A}{d_{1}}+\frac{B}{d_{2}}+\ldots+\frac{N}{d_{i}}\right),
\end{gathered}
$$

where $d_{i}$ is the characterizing size of the grain (in case of spherical grain - the diameter), $S_{i}$ is the absorption surface created by it, and $m_{a m}$ and $\rho_{a m}$ are the mass of the ameliorant and the density of substance, respectively.

Thus, all components of the ameliorant having $(1,2, \ldots, i)$ grain sizes create, respectively, absorption surfaces $S_{1}, S_{2}$, ..., $S_{i}$, while jointly, $S_{a m}=\sum_{i} S_{i}$ efficient surface.

All atoms of any heavy metal (one chemical element) with content of $m_{n}$ in soil, together, create in the environment a

$$
S=\sum_{n} S_{n}=\frac{3 \sum_{n} m_{n}}{2 r_{n} \rho_{n}}
$$

contact surface, where $m_{n}$ is the content of the particular polluting element in soil (the difference between the amount of the mobile forms of heavy metal and the maximum allowable density (MAD) for that element), $\rho_{n}$ is the density of that element, and $r_{n}$ is the radius of the atom (acting ionic field) (Tamoyan, 2013).

We should accept the minimum absorption area created by the ameliorant equal to the total area (optimal indicator) created by the atoms of heavy metals in the soil medium: $S_{a m}=S$. In that case the alignment of (1) and (2) expressions will give:

$$
m_{a m} \cdot\left(\frac{A}{d_{1}}+\frac{B}{d_{2}}+\ldots+\frac{N}{d_{i}}\right)=\rho_{a m} \frac{\sum_{n} m_{n}}{2 r_{n} \rho_{n}} .
$$

From this equation we can calculate the optimal mass of the ameliorant that is the sum of fractions of different sizes: $d_{1}, d_{2}, \ldots, d_{i}$ :

$$
m_{a m}=\rho_{a m} \frac{\sum_{n} m_{n}}{2 r_{n} \rho_{n}} \cdot\left(\frac{A}{d_{1}}+\frac{B}{d_{2}}+\ldots+\frac{N}{d_{i}}\right) .
$$

This expression derived for a single element (heavy metal), in case of availability of different elements in the soil, should be added also according to the composition of those elements.

The proposed method needs to be further improved according to other characteristics of soil's physicochemical absorption.

\section{Conclusion}

To determine the optimal amount of ameliorant (zeolite, 
dacite tuff) used to neutralize heavy metals, the following should be calculated:

a) the shares of the main fractions in the ameliorant;

b) the polluting amounts (the level of soil pollution) of heavy metals;

c) the composition of polluting heavy metals.

According to these indicators, it is possible to calculate the optimal mass of the ameliorant entered into the soil, i.e. the ecoligically substantiated share by expression (4).

\section{References}

1. Amirjanyan, Zh.A. (1993). Microelements in the Soil of RA and Efficiency of Applying Microfertilizers. Abstract of Doctoral Dissertation. M. (in Russian).

2. Galstyan, M.H., Shirinyan, A.V., Harutyunyan, S.S., Tamoyan, S.J., Sargsyan, K.Sh. (2015). Assessment and Remediation of Soils Previously Buried under Mine Tailings and Contaminated with Heavy Metals // Annals of Agrarian Science, V. 13, - N 1.
3. Hayrapetyan, E.M., Galstyan, M.H., Harutyunyan, S.S., Tamoyan, S.J. (2008). Impact of Combined Use of Natural Ameliorants and Organic Fertilizers on the YieldingCapacityofAgriculturalCropsand Accumulation of Heavy Metals in Plants of Technogenically Polluted Soils // Proceedings of the International Conference "Ecological Problems of Agriculture" Yerevan, ASAU, pp. 145-151 (in Russian).

4. Hayrapetyan, E.M., Galstyan, M.H., Harutyunyan, S.S., Tamoyan, S.J. (2009). Impact of Different Technologies on the Yielding Capacity of Tomato and Accumulation of Heavy Metals in Fruits of Technogenically Polluted Soils // Proceedings of the International Conference "Ecological Problems of Agriculture" Yerevan, ASAU, - pp. 148-152 (in Russian).

5. Tamoyan, S.J. (2013). Calculation of Optimization of the Share, Grain Size of the Ameliorant Absorbing Heavy Metals in Soil and the Level of Soil Pollution // Crisis Management and Technologies, Yerevan, - N 1(6) (in Armenian). 